\title{
Tip of the iceberg: patient safety incidents in primary care
}

\author{
Urmimala Sarkar
}

Correspondence to Associate Professor Urmimala Sarkar, Division of General Internal Medicine, University of California, San Francisco, Center for Vulnerable Populations, San Francisco General Hospital, 1001 Potrero Avenue, Building 10, 3rd floor, San Francisco CA 94110, USA;

Urmimala.Sarkar@ucsf.edu

Accepted 13 November 2015 Published Online First 16 December 2015

\section{SLinked}

http://dx.doi.org/10.1136/ bmjqs-2015-004178

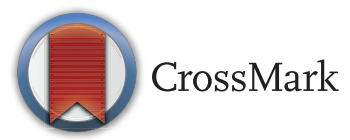

To cite: Sarkar U. BMJ Qual Saf 2016;25:477-479.
Primary care in the US model is fraught with safety hazards. Visits are brief and infrequent, patients are largely selfmanaging, often with multiple comorbid conditions, the extent of healthcare teams varies widely, and the fragmentation of health systems and lack of interoperability among electronic health records (EHRs) means that primary care providers may not have timely, accurate data about patients. ${ }^{1}$ Despite these multiple vulnerabilities, significant gaps remain in our understanding of the safety of primary care. ${ }^{2-4}$

The patient safety movement began in acute-care settings, where adverse events resulting from medical care are more immediately apparent. Even though primary care is the cornerstone of healthcare delivery, relatively less is known about the epidemiology of adverse events in primary care settings. ${ }^{5}$ In an effort to address this gap, Panesar $e t a l^{6}$ conducted a systematic review of patient safety incidents in primary care.

This paper makes a major contribution to the field by providing an overview of the burden of patient safety incidents in primary care. They found that patient safety incidents are slightly less common in primary care, around $2 \%-3 \%$ of visits, compared with approximately $10 \%$ of hospitalisations. ${ }^{7-9}$ Given how much larger and healthier the populations using primary care are compared with hospitalised patients, the frequency of safety incidents in primary care is staggeringly high. Fortunately, only a small proportion of these incidents result in severe harm.

This systematic review highlights multiple challenges in studying the safety of primary care. First, even more than a decade into the patient safety movement, definitional challenges remained. Panesar et $a l^{6}$ defined patient safety incident as 'any unintended or unexpected incident that could have or were judged to have led to patient harm'. Within this broad definition, however, the authors chose to include only incidents of commission rather than omission. In effect, they counted events where the wrong thing was done, but did not count events where the right thing was not done. The fast pace and frequent interruptions associated with primary care are known to lead to errors of omission, ${ }^{10}$ and omissions are a major culprit in missed and delayed diagnoses, ${ }^{11}$ which Panesar et al found to be among the most harmful of primary care safety incidents. Therefore, this analysis likely shows us only the tip of the iceberg. The included studies under-represent the frequency of patient safety incidents, and may especially undercount diagnostic errors. It is critical that, going forward, safety surveillance efforts in primary care include incidents involving errors of both commission and omission.

The ascertainment methods for patient safety incidents require further examination. ${ }^{12}$ Most of these studies employed record review, while other used incident reporting systems or surveys. Each of these ascertainment methods has limitations. Record review leads to lower estimates of incidents because of suboptimal documentation ${ }^{13}$; incident reporting systems are underused, ${ }^{14}$ particularly by physicians ${ }^{15}$ and surveys can include incidents which are not related to safety per se. ${ }^{16}$ Future studies should employ multiple ascertainment methods for primary care patient safety incidents and contrast the resulting estimates.

Evaluating the extent of harm to primary care patients remains a thorny issue. Expert record review does not always yield agreement about harm. ${ }^{17}$ While the authors used a clear definition from the UK National Patient Safety Agency, ${ }^{18}$ the variation in the underlying studies suggests that the definition may not have been applied consistently. One example of an incident without harm is 
an illegible handwritten prescription which does not impact a patient-perhaps because the pharmacist contacted the physician to clarify. However, an erroneous/harmful medication prescribed by a physician but identified and intercepted by a pharmacist, therefore, not reaching the patient, also represents an incident without harm. These two incidents are conceptually distinct. Developing effective interventions requires precision in describing harm.

Many things can go wrong in primary care. The types of incidents that emerged from this synthesis are medication events, diagnostic errors and communication failures, which I infer includes handoffs among outpatient providers. These results underscore the importance of these three areas as the three pillars of primary care safety. All require substantive investment in improvement and evaluation. While electronic prescribing has eliminated certain medication-related incidents, ${ }^{19}$ abundant evidence indicates that medication use remains a locus of safety problems in primary care. $^{20}$ Regarding diagnosis, a recent Institute of Medicine report estimated that every American will experience a missed or delayed diagnosis. ${ }^{21} 22$ The frequency of lasting harm in delayed or missed diagnoses adds to the concern about addressing this often-overlooked safety problem.

Communication failures likely encompass two critical areas for the safety of primary care-handoffs between outpatient providers, who rarely share EHR systems or processes, ${ }^{23}$ and communication with patients. Because patients with chronic conditions are often expected to perform complex self-management tasks independently, ${ }^{24}$ suboptimal communication with patients puts them at risk of harm. ${ }^{25}$ These incidents demonstrate the need to embed effective, health literacy and language-appropriate communication in routine primary care. ${ }^{26-28}$

Panesar et $a l^{6}$ synthesis of patient safety incident rates illuminates the agenda for patient safety in primary care. The field urgently needs consensus around definitions of events, harm and preventability that can be implemented with consistency across multiple settings. These definitions should extend from research to practice. We need multicentre, prospective, epidemiological studies using multiple surveillance methods in order to clearly understand the incidence and prevalence of safety incidents in primary care, including both omission and commission events. Improving communication requires a heightened focus on patient engagement and communication needs and preferences. Interventions that seek to reduce harm to patients should focus on both prevention of adverse drug events and improving diagnosis. This research agenda cannot proceed without substantial investment in primary care patient safety research and quality improvement efforts. This investment should come from health systems, payers and research funders in order to improve safety for all populations receiving primary care.

Because most primary care safety incidents do not lead to immediate harm, they do not seem to carry the same urgency as adverse events in the hospital. But, even if the consequences of a missed cancer diagnosis or dangerous medication combination error take months to years to manifest, they remain just as morbid and just as important to patients as any preventable adverse event in the hospital. Even if most patient safety incidents in primary care carry a low potential for lasting harm, as Panesar et al show, the sheer volume of service delivery translates into a substantial public health burden from patient safety incidents in primary care. The time has thus come to broaden our focus in patient safety accordingly. The tip of the iceberg is a place to start.

Competing interests None declared.

Provenance and peer review Commissioned; internally peer reviewed.

\section{REFERENCES}

1 Sarkar U, Wachter RM, Schroeder SA, et al. Refocusing the lens: patient safety in ambulatory chronic disease care. $J t$ Comm J Qual Pat Saf 2009;35:377-83, 41.

2 Gandhi TK, Lee TH. Patient safety beyond the hospital. N Engl J Med 2010;363:1001-3.

3 Wachter RM. Is ambulatory patient safety just like hospital safety, only without the "stat"? Ann Intern Med 2006;145:547-9.

4 Smith PC, Araya-Guerra R, Bublitz C, et al. Missing clinical information during primary care visits. JAMA 2005;293:565-71.

5 The Safer Primary Care Expert Working Group. Safer primary care: a global challenge. Geneva: World Health Organization, 2012. http://www.who.int/patientsafety/summary_report_of_ primary_care_consultation.pdf (accessed 23 Oct 2015).

6 Panesar S, de $\bar{S}$ ilva D, Carson-Stevens A, et al. How safe is primary care? A systematic review. BMJ Qual Saf 2016;25:544-53.

7 Vincent C, Neale G, Woloshynowych M. Adverse events in British hospitals: preliminary retrospective record review. $B M J$ 2001;322:517-9.

8 Makeham M, Dovey S, Runciman W, Larizgoitia I. Methods and Measures used in Primary Care Patient Safety Research. Better knowledge for safer care. World Health Organization, 2008.

9 To Err Is Human: Building a Safer Health System. Washington, DC: National Academy Press, 2000.

10 Linzer M, Manwell LB, Williams ES, et al. Working conditions in primary care: physician reactions and care quality. Ann Intern Med 2009;151:28-36, W6-9.

11 Singh H, Giardina TD, Meyer AN, et al. Types and origins of diagnostic errors in primary care settings. JAMA Intern Med 2013;173:418-25.

12 Detection of Safety Hazards. Secondary Detection of Safety Hazards [Webpage] 2015. https://psnet.ahrq.gov/primers/ primer/24/detection-of-safety-hazards (accessed 23 Oct 2015).

13 Gandhi TK, Burstin HR, Cook EF, et al. Drug complications in outpatients. J Gen Intern Med 2000;15:149-54. 
14 Cullen DJ, Bates DW, Small SD, et al. The incident reporting system does not detect adverse drug events: a problem for quality improvement. Jt Comm J Qual Improv 1995; 21:541-8.

15 Rowin EJ, Lucier D, Pauker SG, et al. Does error and adverse event reporting by physicians and nurses differ? Jt Comm J Qual Pat Saf 2008;34:537-45.

16 Kuzel AJ, Woolf SH, Gilchrist VJ, et al. Patient reports of preventable problems and harms in primary health care. Ann Fam Med 2004;2:333-40.

17 Gandhi TK, Kachalia A, Thomas EJ, et al. Missed and delayed diagnoses in the ambulatory setting: a study of closed malpractice claims. Ann Intern Med 2006;145:488-96.

18 Seven steps to patient safety: The Full Reference Guide. Second ed. London: National Patient Safety Agency, 2004.

19 Kaushal R, Kern LM, Barrón Y, et al. Electronic prescribing improves medication safety in community-based office practices. J Gen Intern Med 2010;25:530-6.

20 Overhage JM, Gandhi TK, Hope C, et al. Ambulatory computerized prescribing and preventable adverse drug events. J Patient Saf 2015. Published Online First: 21 May 2015.

21 Institute of Medicine. Improving Diagnosis in Health Care. Washington, DC: The National Academies Press, 2015.
22 Singh H, Meyer AN, Thomas EJ. The frequency of diagnostic errors in outpatient care: estimations from three large observational studies involving US adult populations. BMJ Qual Saf 2014;23:727-31.

23 van Walraven C, Taljaard M, Bell CM, et al. Information exchange among physicians caring for the same patient in the community. CMAJ 2008;179:1013-8.

24 Buetow S, Elwyn G. Patient safety and patient error. Lancet 2007;369:158-61.

25 Lemer C, Bates DW, Yoon C, et al. The role of advice in medication administration errors in the pediatric ambulatory setting. J Patient Saf 2009;5:168-75.

26 Persell SD, Osborn CY, Richard R, et al. Limited health literacy is a barrier to medication reconciliation in ambulatory care. J Gen Intern Med 2007;22:1523-6.

27 Davis TC, Wolf MS, Bass PF III, et al. Literacy and misunderstanding prescription drug labels. Ann Intern Med 2006;145:887-94.

28 Bradshaw M, Tomany-Korman S, Flores G. Language barriers to prescriptions for patients with limited English proficiency: a survey of pharmacies. Pediatrics 2007;120: e225-35. 